Revue d'histoire de l'Amérique française

DEV REVUE D.HISTOIRE DE L'AMÉRIQUE FRANÇAISE

\title{
Le militantisme des travailleurs au Québec et en Ontario, niveau de syndicalisation et mouvement de grèves (1900-1980)
}

\section{Jacques Rouillard}

Volume 37, numéro 2, septembre 1983

Travailleurs et mouvements sociaux

URI : https://id.erudit.org/iderudit/304154ar

DOI : https://doi.org/10.7202/304154ar

Aller au sommaire du numéro

Éditeur(s)

Institut d'histoire de l'Amérique française

ISSN

0035-2357 (imprimé)

1492-1383 (numérique)

Découvrir la revue

\section{Citer cet article}

Rouillard, J. (1983). Le militantisme des travailleurs au Québec et en Ontario, niveau de syndicalisation et mouvement de grèves (1900-1980). Revue d'histoire de l'Amérique française, 37(2), 201-225. https://doi.org/10.7202/304154ar d'utilisation que vous pouvez consulter en ligne. 


\title{
LE MILITANTISME DES TRAVAILLEURS AU QUÉBEC ET EN ONTARIO NIVEAU DE SYNDICALISATION ET MOUVEMENT DE GREVES (1900-1980)
}

\author{
JACQUES ROUILLARD \\ Université de Montréal
}

La lecture du bilan historiographique de Desmond Morton dans les pages de ce numéro de la Revue pourra amener plus d'un lecteur à s'interroger sur la situation de l'histoire ouvrière au Québec. Malheureusement, nos connaissances sur les travailleurs québécois accusent un retard considérable lorsqu'on trace un parallèle avec l'historiographie canadienne-anglaise. L'histoire des institutions mises en place par les travailleurs anglophones (syndicats, organisations politiques, coopératives, etc.) est relativement bien connue et de nouveaux courants historiographiques cherchent à dégager les traits culturels de la classe ouvrière canadienne-anglaise.

La première synthèse véritable du syndicalisme canadien, celle de H.A. Logan ${ }^{1}$, a paru en 1928 alors qu'on ne peut trouver un ouvrage similaire sur le syndicalisme québécois (Histoire du mouvement ouvrier au Québec (1825-1976)2 que depuis à peine quatre ans. Et encore, ce dernier volume comporte de nombreuses faiblesses car les auteurs n'ont pu appuyer leur synthèse que sur de rares monographies et sur des ouvrages à caractère superficiel. En fait, l'histoire d'une institution aussi fondamentale pour les travailleurs que le mouvement syndical reste encore à faire au Québec. On ne doit pas alors s'étonner que les nouvelles problématiques en histoire de la culture des travailleurs qui ont vu le jour en Grande-Bretagne, aux États-Unis et par ricochet au Canada anglais n'aient pas encore trouvé d'écho chez les historiens québécois ${ }^{3}$.

1 H.A. Logan, The History of Trade Union Organizations in Canada (Chicago, University of Chicago Press, 1928), $427 \mathrm{p}$

2 CSN-CEQ, Histoire du mouvement ouvrier au Québec (1825-1976) (Montréal, CSNCEQ, 1979), $235 \mathrm{p}$.

3 Yvan Lamonde et ses collaborateurs ont dressé un bilan historiographique des travaux réalisés sur la culture ouvrière à Montréal de 1880 à 1920 . Aucun ne s'inscrit dans la problématique définie par E.P. Thompson, H.G. Gutman et D. Montgomery.

On relève toutefois des travaux riches d'informations sur la culture ouvrière: les excellents articles de Bettina Bradbury qui, en s'inspirant de l'historiographie américaine, a mis à profit les

[201]

RHAF, vol. 37, no 2, septembre 1983 
Bien que ces travaux posent de nouvelles questions fort pertinentes au passé, il n'en reste pas moins qu'ils profitent du résultat des recherches entreprises par leurs devanciers. Un E.P. Thompson, par exemple, n'aurait pu écrire son étude magistrale de la classe ouvrière anglaise ${ }^{4}$ sans l'apport de trois générations d'historiens anglais spécialisés en histoire sociale. De même, aux États-Unis, un David Montgomery peut tracer les étapes du développement de la conscience ouvrière américaine ${ }^{5}$ en s'appuyant sur des études nombreuses du mouvement ouvrier américain. L'abondance de travaux sur une question permet un foisonnement des hypothèses et un renouvellement des problématiques.

Dans l'excellent bilan qu'il trace de l'historiographie des travailleurs québécois ${ }^{6}$, Fernand Harvey s'interroge sur le retard des chercheurs à s'intéresser à l'histoire ouvrière. Il relève à juste titre que pendant longtemps les chercheurs ont réservé l'initiative historique à la bourgeoisie ${ }^{7}$; c'est pourquoi leurs travaux ont plutôt été orientés vers l'étude de ce groupe social. Les travailleurs sont alors marginalisés ou carrément absents de l'histoire de notre société.

La génération d'intellectuels québécois d'après-guerre découvre l'existence de la classe ouvrière, mais elle n'en fait un élément significatif de l'histoire du Québec qu'après la Deuxième Guerre. Pour les périodes antérieures, elle imagine une société rurale dominée par le clergé auquel est inféodée la petite bourgeoisie francophone. Selon cette interprétation, l'industrialisation n'atteint véritablement le Québec qu'avec la Deuxième Guerre, ce qui aurait pour effet d'ébranler les fondements de la société traditionnelle. Les sociologues Jacques Dofny et Marcel Rioux vont même jusqu'à affirmer que la conscience ethnique qui s'est affirmée au Canada français depuis le milieu du XIX ${ }^{\mathrm{e}}$ siècle a retardé la prise de conscience des classes sociales ${ }^{8}$. Ils visent en parti-

recensements manuscrits et l'ouvrage de 'lerry Copp, Classé ouvrière et pauvreté, d'abord publié en anglais en 1974, qui n'a rien à envier aux ouvrages plus récents en histoire de la culture ouvrière. (Y. Lamonde, L. Ferretti et D. Leblanc, La culture ouvrière à Montréal (1880-1920): bilan historiographique (Québec, I.Q.R.C., 1982), 176 p.; Bettina Bradbury, "L'économie familiale et le travail dans une ville en voie d'industrialisation: Montréal dans les années 1870», dans N. Fahmy-Eid et M. Dumont, Maîtresses de maison, maîtresses d'école (Montréal, Boréal Express, 1983), 287-318, et «Pigs, Cows and Boarders, Non-Wage Forms of Survival among Montreal Families 1861-1891», Communication à la Société historique du Canada, Vancouver, juin 1983, 37 p.; Terry Copp, Classe ouvrière et pauvreté. Les conditions de vie des travailleurs montréalais 1897-1929 (Montréal, Boréal Express, 1978), 213 p.).

4 E. P. Thompson, The Making of the English Working Class (Harmondsworth, Penguin Books, 1968), $958 \mathrm{p}$.

5 David Montgomery, Worker's control in America (Cambridge, Cambridge University Press, 1979), $189 \mathrm{p}$. 48.

Fernand Harvey, Le mouvement ouvrier au Québec (Montréal, Boréal Express, 1980), 9-

7 Ibid., 10-12.

8 Jacques Dofny et Marcel Rioux, «Les classes sociales au Canada français», dans M. Rioux et Y. Martin, La société canadienne-française (Montréal, Hurtubise, 1971), 315-324. Et Marcel Rioux, «Conscience ethnique et conscience de classe», Recherches sociographiques, VI, I (1965). 
culier la classe ouvrière francophone qui réagit dans les moments de crise nationale, mais qui demeure muette devant le développement du capitalisme. «Le syndicalisme, affirment-ils, fut lent à démarrer au Québec. Dans ce domaine comme dans bien d'autres, les intérêts de la classe ouvrière furent aliénés au profit de ceux du groupe ethnique considéré comme valeur suprême.»"

Ils appuient leur conclusion sur l'ouvrage édité par Pierre-Elliott Trudeau sur la grève de l'amiante de $1949^{10}$. Ce volume écrit en collaboration représente la première étude fouillée sur un épisode de l'histoire ouvrière. Il est basé sur des recherches sérieuses et constitue un modèle d'analyse d'une grève. La plupart des collaborateurs partagent le point de vue que la grève de 1949 constitue un point tournant dans l'histoire sociale du Québec. C'est d'ailleurs pourquoi, sept ans après l'événement, ils consacrent une étude aussi complète à cette grève. Les auteurs se sont bien documentés sur le conflit lui-même, mais quand vient le temps pour certains d'entre eux de le situer dans un contexte plus large, la pauvreté des connaissances en histoire sociale et syndicale les conduit à des exagérations et à des erreurs d'interprétation. Désireux de montrer l'importance du conflit, ils ont tendance à amplifier les enjeux et l'impact de la grève et à accentuer le conservatisme de l'histoire des décennies antérieures ${ }^{11}$.

Ils commencent d'abord par identifier le syndicalisme au Canada français avec le syndicalisme catholique qui serait issu d'une réaction nationaliste et qui n'aurait pas mené une action syndicale efficace depuis sa fondation jusqu'à 1945. «Le syndicalisme autochtone, écrit Gilles Beausoleil, avait été accepté dans le milieu canadien-français à condition de ne pas introduire des objectifs sociaux différents des objectifs de la communauté.» ${ }^{12}$ «Jusqu'à la grève de l'amiante, renchérit JeanCharles Falardeau, tout différend ouvrier d'une certaine envergure auquel le syndicalisme confessionnel se trouvait mêlé était, à son point d'origine, défini, et à son point d'arrivée, réglé par la trinité patronatgouvernement-Église ... ${ }^{13}$ » Pour la première fois à l'occasion de cette grève, conclut P.-E. Trudeau, «le mouvement ouvrier uni n'a à reculer devant aucune conjonction de forces ${ }^{14}{ }^{14}$; il représente de la sorte une puissance nouvelle qui annonce l'avènement de temps nouveau ${ }^{15}$. Selon eux, la classe ouvrière vient ainsi d'affirmer une présence autonome dans la société canadienne-française.

\footnotetext{
9 J. Dofny et M. Rioux, op. cit., 318.

10 Pierre Elliott Trudeau (éd.), La Grève de l'amiante (Montréal, Éditions du Jour, 1970), $430 \mathrm{p}$.

Ibid., 31 et 361 .

Ibid., 209.

Ibid., XIV

Ibid., 392.

Ibid., 379.
} 
Cette interprétation de l'histoire du mouvement ouvrier est demeurée dominante jusqu'à nos jours. On la retrouve dans la plupart des articles et volumes consacrés à l'histoire ouvrière ${ }^{16}$, et partant, dans les synthèses d'histoire du Québec. Nous verrons dans les pages qui suivent que la classe ouvrière s'est manifestée bien avant la grève de l'amiante et que le niveau de militantisme atteint au Québec n'a rien à envier, par exemple, à celui des travailleurs ontariens.

\section{La notion de militantisme}

Le militantisme ouvrier renvoie à la notion de solidarité et de combativité des travailleurs. La solidarité s'exprime quand des travailleurs prennent conscience de leur communauté d'intérêts et de la nécessité de s'unir pour défendre collectivement ces intérêts. La combativité résulte des énergies déployées à la promotion de la cause ouvrière. Bien sûr, le mouvement syndical témoigne de la solidarité des travailleurs, mais c'est loin d'être la seule forme d'action collective en milieu ouvrier. On en retrouve également l'expression dans les sociétés de secours mutuels qui ont fleuri dans la deuxième moitié du $\mathrm{XX}^{\mathrm{e}}$ siècle et à l'intérieur du mouvement coopératif urbain. Cette solidarité se réflète aussi parmi les associations de quartier, les clubs ouvriers et dans l'action politique ouvrière. Il est possible également qu'elle transparaisse ailleurs comme dans les associations religieuses des paroisses ouvrières ou dans le populisme politique sur la scène municipale montréalaise. Mais comme la recherche en histoire ouvrière est encore jeune, nos connaissances sur ces phénomènes demeurent bien limitées. Il est alors difficile d'évaluer l'ampleur de la solidarité ouvrière dans ces manifestations et de déterminer le degré d'affirmation des travailleurs québécois.

Par contre, certaines sources permettent de lever le voile sur la solidarité syndicale et la combativité des travailleurs dans l'action de grève. En effet, les statistiques que nous avons recueillies nous permettent d'évaluer avec une marge d'erreur raisonnable le niveau de syndicalisation et le mouvement de grèves. Nous tracerons donc l'évolution de ces deux variables depuis le début du siècle en faisant ressortir les principaux éléments d'explication. De plus, nous mettrons en relation nos données pour le Québec avec celles de l'Ontario afin de pouvoir comparer le degré de militantisme des travailleurs dans chaque province.

16 Par exemple: CSN-CEQ, Histoire du mouvement ouvrier..., op. cit.; Louis-Marie Tremblay, Le syndicalisme québécois: idéologies de la CSN et de la FTQ (1940-1970) (Montréal, PUM, 1972), 286 p.; Desmond Morton et Terry Copp, Working People. An Illustrated History of Canadian Labour (Ottawa, Deneau and Greenberg, 1980), 349 p. 


\section{I) Le niveau de syndicalisation}

On ne peut ignorer l'importance du mouvement syndical dans l'affirmation des travailleurs: "Le syndicalisme, écrit Guy Caire, est l'incarnation de la classe ouvrière. ${ }^{17}$ Effectivement, au Québec, le syndicalisme a joué un rôle central dans l'évolution de la classe ouvrière. C'est à travers cette institution que les travailleurs se sont manifestés avec le plus de force et de la façon la plus autonome.

Il est une source éclairante sur la syndicalisation dont on n'a pas encore exploité la richesse. Il s'agit des données statistiques recueillies par le ministère fédéral du Travail ${ }^{18}$. Dans une publication intitulée Rapport annuel sur les associations ouvrières au Canada (l'appellation a varié au cours des années), les fonctionnaires du ministère ont compilé des statistiques sur le nombre de syndicats, les effectifs syndicaux et l'affiliation syndicale. Pour une longue période, la brochure comprend des ventilations par province et par ville.

Cependant, cette source appelle des réserves et doit être utilisée avec précaution. Nous présentons en annexe une critique de ces données statistiques et précisons la méthodologie que nous avons utilisée. Même si ces données présentent des lacunes, elles permettent d'établir approximativement l'évolution du syndicalisme au Québec et de tirer des conclusions pertinentes sur le degré de solidarité syndicale au Québec.

\section{a) Période 1901-1941}

Les premiers syndicats dans la province sont apparus au début du $\mathrm{XIX}^{\mathrm{e}}$ siècle et le mouvement syndical devient une force significative à Montréal et à Québec dès les années 1880. Les syndiqués se donnent alors des structures d'intervention aux niveaux politiques municipal, provincial et fédéral. Au tournant du siècle, la poussée du syndicalisme international amène l'éclosion de très nombreux syndicats d'ouvriers de métier, et partant une augmentation importante des effectifs syndicaux ${ }^{19}$. Les «internationaux» qui jouissent d'une base solide aux Etats-Unis profitent du contexte économique favorable qui prévaut pendant la première décennie du $\mathrm{XX}^{\mathrm{e}}$ siècle.

17 Guy Caire, Les syndicats ouvriers (Paris, PUF, 1971), 17.

18 Il y a une exception: Hélène David dans sa thèse de doctorat nous présente un tableau sur le taux de syndicalisation au Québec de 1931 à 1968 à partir de cette source. Elle a effectué des calculs pour obtenir un estimé annuel des effectifs syndicaux et les taux de syndicalisation en fonction de la main-d'oeuvre non agricole rémunérée. Son travail de pionnier est fort valable. Notre étude se différencie de sa recherche en ce que nous remontons plus loin dans le temps, faisons des comparaisons avec l'Ontario et interprétons l'évolution de la syndicalisation au Québec (Hélène David, L'activité de grève au Québec de 1945 à 1967: une analyse sociologique, Thèse de doctorat, Paris, École Pratique des Hautes Études, 1975, appendice B. tableau 6-A. Le tableau est reproduit à la fin d'un de ses articles dans Fernand Harvey, op. cit., 288). 1979), 119.

9 Voir J. Rouillard, Les syndicats nationaux au Québec de 1900-1930 (Québec, PUL, 
Pour les années subséquentes, le taux de syndicalisation se présente ainsi:

\begin{tabular}{|c|c|c|c|c|}
\hline \multicolumn{5}{|c|}{ TABLEAU I } \\
\hline & $\begin{array}{c}\text { Nombre de } \\
\text { syndicats }\end{array}$ & $\begin{array}{c}\text { Effectifs } \\
\text { déclarés }\end{array}$ & $\begin{array}{c}\text { Effectifs } \\
\text { estimés }\end{array}$ & $\begin{array}{c}\text { Pourcentage des } \\
\text { effectifs estimés } \\
\text { par rapport à la } \\
\text { population active } \\
\text { non agricole }\end{array}$ \\
\hline & & & & 5,6 \\
1911 & 228 & 13868 & 25200 & 17,3 \\
1916 & 332 & 30300 & 46800 & 9,0 \\
1921 & 492 & 52060 & 97800 & 16,8 \\
1931 & 460 & 52786 & 75800 & 72100 \\
1936 & 501 & 58620 & 89500 & 157700 \\
1941 & 573 & 74572 & 121280 & 157 (1911-1941) \\
\hline
\end{tabular}

Selon nos estimations, le nombre de syndiqués vient bien près de doubler entre 1911 et 1916, et ce, à la fois grâce à l'expansion du syndicalisme international et à la création de syndicats catholiques dans divers diocèses de la province.

La Première Guerre, du moins à ses débuts, ne se traduit pas par une extension du syndicalisme; il faut attendre 1918 et surtout 1919 , 1920 et 1921 pour voir s'affirmer le mouvement syndical. Il tire avantage du climat de prospérité qui se poursuit au-delà du conflit, jusqu'à ce que les pays européens se rétablissent définitivement. La forte poussée inflationniste née de la guerre crée également un contexte favorable à la syndicalisation car les travailleurs cherchent à protéger leur pouvoir d'achat. En plus, ces années sont marquées par une effervescence ouvrière sans précédent dans les pays occidentaux. Après une guerre contre des régimes despotiques, les milieux ouvriers manifestent le désir de réformer la société en profondeur.

L'année 1921, qui coïncide avec la naissance de la Confédération des travailleurs catholiques du Canada, constitue un sommet de la progression syndicale. C'est une année exceptionnelle qui n'est pas représentative cependant des années vingt; elle a donné lieu à une poussée

20 Notre estimation des effectifs tient compte de plusieurs variables que nous analysons en annexe. Pour les effectifs syndicaux: Canada, Department of Labour, Report on Labour Organizations in Canada (1911): 104; 251; (1931): 239 et 243; (1936): 204 et 207; (1941): 9 et 16. Pour la population active non agricole: B.F.S. Tendances occupationnelles au Canada 1901-1941, Bulletin no 0-6, Recensement du Canada, 1941 (98-1941, m 33): 10 et 11. 
bien temporaire du syndicalisme et à des adhésions bien fragiles surtout chez les syndicats catholiques.

Après le plateau atteint en 1921 , le mouvement syndical régresse au cours des cinq années suivantes avec la récession économique de 1921. La reprise industrielle de 1925 à 1929 se traduit par une croissance syndicale. En 1931, au moment où la crise économique de 1929 se répercute sur les effectifs syndicaux, $9 \%$ de la population active non agricole profitent des bienfaits de la syndicalisation. Ce sont en grande majorité des ouvriers de métier dans la construction, le transport ferroviaire, la métallurgie (machinistes, mouleurs) et l'industrie du papier. Le mouvement syndical ne rejoint pas encore véritablement la grande industrie manufacturière où on retrouve les concentrations les plus fortes de travailleurs: textile, pâte et papier, matériel de transport, vêtement (syndiqué partiellement), etc. La main-d'oeuvre s'accroît dans ces industries alors que les effectifs syndicaux demeurent à peu près stables de 1921 à 1931. C'est ce qui explique que le taux de syndicalisation régresse pendant ces dix années.

Puis viennent s'ajouter les effets de la dépression de 1929 où les syndicats perdent le tiers de leurs effectifs (1929-1933). Comme pour les périodes antérieures, la reprise amorcée en 1936 a des effets bénéfiques sur la syndicalisation. Et, chose surprenante, c'est la Confédération des travailleurs catholiques du Canada qui profite jusqu'à la guerre de ce contexte favorable. Elle est largement responsable de la progression du taux de syndicalisation de 9 à $16,4 \%$ pendant les années trente. Son expansion s'est réalisée parmi les ouvriers moins qualifiés dans la grande industrie: textile, vêtement, bois, papier et métallurgie. La souplesse de ses structures, son implantation dans les régions et le recours à la loi d'extension juridique des conventions collectives (1934) lui valent cet afflux de syndiqués alors que les «internationaux» conservent des effectifs stationnaires ${ }^{21}$. Durant la guerre par contre, ils lui disputeront l'adhésion des nouveaux syndiqués avec beaucoup plus de succès.

\section{b) Période 1941-1976}

Le mouvement syndical au Québec prend un essor énorme durant la Deuxième Guerre, croissance qui se ralentit quelque peu par la suite. La forte demande de main-d'oeuvre pendant la guerre permet un meilleur pouvoir de négociation des travailleurs, d'où leur intérêt à joindre les associations syndicales. L'appui donné à la syndicalisation par les deux niveaux de gouvernement grâce à de nouvelles législations du travail contribue également à l'essor du syndicalisme.

21 J. Rouillard, Histoire de la CSN (1921-1981) (Montréal, CSN/Boréal Express, 1981), 113-115. 


\begin{tabular}{|c|c|c|c|c|}
\hline \multicolumn{5}{|c|}{ TABLEAU II } \\
\hline & $\begin{array}{c}\text { Nombre de } \\
\text { syndicats }\end{array}$ & $\begin{array}{c}\text { Effectifs } \\
\text { déclarés }\end{array}$ & $\begin{array}{c}\text { Effectifs } \\
\text { estimés }\end{array}$ & $\begin{array}{c}\text { Pourcentage des } \\
\text { effectifs estimés } \\
\text { par rapport } \\
\text { aux salariés }\end{array}$ \\
\hline & & & & 20,7 \\
1941 & 671 & 121280 & 157700 & 29,3 \\
1946 & 989 & 208546 & 259700 & 26,5 \\
1951 & 1126 & 239800 & 279800 & 28,1 \\
1956 & 1367 & 316682 & 352500 & 30,5 \\
1961 & 1606 & 353300 & 399800 & 35,7 \\
1966 & & 591551 & & 39,3 \\
1970 & & 704413 & & 37,0 \\
1976 & & 846619 & & 36,7 \\
1977 & & 773282 & & (1941-1977) \\
\hline
\end{tabular}

Après le conflit mondial, les effectifs syndicaux augmentent au même rythme que la main-d'oeuvre si bien que le taux de syndicalisation demeure à peu près identique jusqu'en 1961. Contrairement à l'idée émise par les collaborateurs du volume La Grève de l'Amiante, les années cinquante ne révèlent pas de progrès marquant du syndicalisme, c'est plutôt la guerre qui lui a donné une impulsion décisive en faisant faire un bond de 64\% aux effectifs syndicaux (1941-1946). Les nouveaux venus au syndicalisme se retrouvent particulièrement dans l'industrie métallurgique, l'alimentation et le secteur des services (commerce, administration publique). La CTCC profite de la syndicalisation de ce dernier secteur alors que les unions internationales affiliées à la $\mathrm{CIO}$ (Congress of Industrial Organizations) dominent dans les deux premiers.

Depuis 1960, l'événement central dans le monde syndical demeure l'organisation des employés du secteur public et parapublic. Commencée depuis la dernière guerre, la syndicalisation de ces employés est devenue presque complète dans le sillage de la Révolution tranquille. C'est pourquoi les effectifs syndicaux totaux s'accroissent de $42,8 \%$ entre 1963 et 1966 et que le taux de syndicalisation passe de $30,1 \%$ à $35,7 \%$ au cours de ces trois années ${ }^{23}$. Dans le secteur privé, il y a lieu

22 Voir l'annexe pour des précisions sur la méthode ayant servi à estimer les effectifs syndicaux. Pour les effectifs syndicaux: Canada, Ministère du Travail, Organisation des travailleurs au Canada (1941): 9; (1946): 13; (1951): 9; (1956): 12; Gazette du travail, mars 1962, 305. Pour 1966 à 1970: François Delorme et G. Lassonde, Aspects de la réalité syndicale québécoise, 1976 (Québec, Ministère du Travail et de la Main-d'oeuvre, 1978), 3. Pour 1977, Peter O’Brien, The Myth of Labour Instability (Halifax Department of Regional Economic Expansion and Nova Scotia Department of Development, 1980), 2. Concernant les travailleurs salariés: BFS, Canadian Labour Estimates (1931-1945): 18; The Labour Force (1945-1955): 98; Estimations du nombre de salariés par province et par industrie, 1961-1976, 30.

${ }_{23}$ Gazette du Travail, janvier 1964, 12; février 1967, 93. 
de penser que le niveau de syndicalisation augmente au même rythme que la main-d'oeuvre pendant les années soixante ${ }^{24}$.

En revanche, à partir de 1975, ce rythme se ralentit sérieusement, si bien que le membership syndical total demeure à peu près stagnant depuis cette date. C'est ce qui explique que le taux global de syndicalisation diminue de 41,3 à 36,7\% de 1974 à 1977, dernière année pour laquelle on possède des statistiques ${ }^{25}$. Il ne serait pas surprenant que ce taux diminue encore plus dans les années subséquentes. La régression du mouvement syndical trouve son explication à deux sources: d'une part, les syndicats ont fait le plein des travailleurs du secteur public et parapublic dont le nombre augmente très peu dans les années 70; en outre, la syndicalisation du secteur privé demeure très ardue surtout en période de difficultés économiques comme celle que nous connaissons depuis 1973-74. Notre historique de la syndicalisation montre qu'en général le mouvement syndical progresse dans les moments de prospérité et régresse avec les récessions économiques. La force revendicative des syndicats diminue en période de dépression et les travailleurs craignent de perdre leur emploi en joignant un syndicat.

\section{c) La syndicalisation en Ontario}

Comme les pages qui précèdent le démontrent, le mouvement de syndicalisation au Québec s'est fait graduellement au cours du siècle. Bien avant la Deuxième Guerre, les syndicats regroupent un nombre significatif de travailleurs. On pourrait penser cependant que cette progression est plus lente qu'ailleurs. Les travailleurs québécois accusentils un retard à prendre conscience de la nécessité de la solidarité syndicale? Pour répondre à cette question, nous n'avons pas trouvé meilleure façon que de comparer le Québec à l'Ontario en utilisant la même source de documentation et la même méthodologie qu'aux deux tableaux précédents. Nous ajoutons à titre d'information des données sur les États-Unis.

Il ressort que le niveau de syndicalisation au Québec est presque continuellement supérieur à celui de la province voisine. En effet, l'Ontario ne devance le Québec qu'au début du siècle et dans les années cinquante, années où pourtant on disait que le syndicalisme québécois avait atteint «la condition adulte».

La supériorité du Québec nous apparaît s'expliquer par la présence du syndicalisme catholique qui rejoint un groupe de travailleurs négligés par les unions internationales. En effet, c'est lorsque les syndicats catholiques s'implantent dans la province que le taux de syndicalisation

24 F. Delorme et G. Lassonde, op. cit., 9 à 11; Québec, Ministère du Travail et de la Maind'oeuvre, Taux du syndicalisme au Québec (1970), 126 p.

25 F. Delorme et G. Lassonde, op. cit., 3; Peter O'Brien, op. cit., 2. 


\begin{tabular}{|c|c|c|c|c|c|c|c|c|}
\hline \multicolumn{9}{|c|}{$\begin{array}{c}\text { TABLEAU III } \\
\text { TAUX DE SYNDICALISATION AU QUÉBEC, } \\
\text { EN ONTARIO ET AUX ÉTATS-UNIS }(1911-1977)^{26}\end{array}$} \\
\hline \multicolumn{3}{|c|}{$\begin{array}{l}\text { Effectifs } \\
\text { estimés ou } \\
\text { déclarés }\end{array}$} & \multicolumn{3}{|c|}{$\begin{array}{l}\text { Pourcentage des } \\
\text { effectifs par rapport à } \\
\text { la population active } \\
\text { non agricole }\end{array}$} & \multicolumn{3}{|c|}{$\begin{array}{l}\text { Pourcentage des } \\
\text { effectifs par rapport } \\
\text { aux salariés }\end{array}$} \\
\hline & Qué. & Ont. & Qué. & Ont. & U.S. & Qué. & Ont. & U.S. \\
\hline 1911 & 25200 & 57800 & 5,6 & 8,4 & 10,0 & & & \\
\hline 1921 & 97800 & 99800 & 17,4 & 12,1 & 17,8 & & & \\
\hline 1931 & 72100 & 85500 & 9,0 & 8,2 & 11,8 & & & \\
\hline 1941 & 157700 & 157900 & 16,8 & 13,3 & 23,0 & 20,7 & 15,3 & \\
\hline 1946 & 259700 & 291900 & 25,3 & 21,1 & 30,4 & 29,3 & 23,4 & \\
\hline 1951 & 279800 & 406500 & 23,3 & 25,1 & 31,3 & 26,5 & 27,5 & \\
\hline 1956 & 352500 & 568700 & 24,3 & 29,1 & 31,3 & 28,1 & 32,6 & \\
\hline 1961 & 399800 & 607300 & 25,3 & 28,3 & 27,9 & 30,5 & 32,1 & 30,2 \\
\hline 1966 & 591551 & 772958 & & & & 35,7 & 35,3 & \\
\hline 1970 & 704413 & 926162 & & & & 39,3 & 36,1 & 30,1 \\
\hline 1977 & 773283 & 1016100 & & & & 36,7 & 30,9 & \\
\hline
\end{tabular}

dépasse celui de l'Ontario. Et leur dynamisme dans les années trente creuse l'écart au point de valoir au Québec un taux de syndicalisation de $35 \%$ supérieur en 1941. La tendance se renverse après la guerre avec la consolidation des syndicats industriels $(\mathrm{CIO})$ à l'ouest de l'Outaouais. Il est possible aussi que l'anti-syndicalisme du gouvernement Duplessis ait ralenti la progression syndicale au Québec au cours de la période.

L'écart redevient favorable à la province francophone qui surpasse même les États-Unis avec la syndicalisation massive du secteur public et parapublic. L'Ontario qui conserve un niveau de syndicalisation supérieur au Québec dans l'industrie manufacturière accuse un retard dans l'organisation des employés du tertiaire public ${ }^{27}$. Ces travailleurs, qui se sont syndiqués plus rapidement et plus complètement au Québec, lui procurent toujours en 1977 un taux global de syndicalisation supérieur à celui de la province voisine.

La comparaison entre ces deux régions du Canada fait clairement ressortir que s'il y a «retard» de la classe ouvrière québécoise, ce n'est pas du côté de l'adhésion syndicale qu'il faut le chercher. En général,

26 Effectifs estimés jusqu'en 1961. Mêmes notes qu'aux tableaux précédents et Leo Troy, Trade Union Membership, 1897-1962, Review of Economics and Statistics, 47 (février 1965), 94; J.K. Eaton, op. cit., 30.

27 J.K. Eaton, op. cit., 114 et 123; Peter O'Brien, op. cit., 5. 
les travailleurs québécois ont été aussi prompts et même plus nombreux à joindre les rangs des syndicats que les travailleurs ontariens. Ils ne sont pas tellement loin non plus derrière les travailleurs étatsuniens. Comment alors pouvoir leur reprocher d'avoir été muets devant le développement du capitalisme industriel.

\section{II) L'affiliation syndicale}

De nombreux travaux ont tendance à identifier le syndicalisme québécois aux seuls syndicats catholiques, accusés jusqu'à la guerre, d'être des syndicats «jaunes» épris davantage d'action catholique que d'action proprement syndicale ${ }^{28}$. Nous avons montré ailleurs que cette interprétation de l'histoire de la CTCC doit être revisée à la lumière d'une meilleure connaissance de ses pratiques syndicales pendant les années vingt et trente ${ }^{29}$. De même, c'est une erreur que d'ignorer la présence au Québec d'autres groupes syndicaux.

$\grave{A}$ aucun moment de son histoire, la centrale catholique ne regroupe la majorité des syndiqués québécois; elle ne constitue pas non plus le principal groupe syndical dans la province. Comme dans l'ensemble de l'Amérique du Nord, ce sont les syndicats affiliés aux unions internationales qui possèdent les effectifs les plus importants au Québec.

\begin{tabular}{|c|c|c|c|c|c|}
\hline \multicolumn{6}{|c|}{$\begin{array}{c}\text { TABLEAU IV } \\
\text { ALLÉGEANCE SYNDICALE AU QUÉBEC }(1901-1976)^{30}\end{array}$} \\
\hline \multicolumn{2}{|c|}{$\begin{array}{c}\text { Nombre de } \\
\text { syndicats } \\
\text { internationaux }\end{array}$} & $\begin{array}{l}\text { Nombre de } \\
\text { syndicats } \\
\text { CTCC/CSN }\end{array}$ & $\begin{array}{c}\text { Autres } \\
\text { syndicats }\end{array}$ & $\begin{array}{c}\text { Total } \\
\text { des } \\
\text { syndicats }\end{array}$ & $\begin{array}{l}\text { Pourcentage des } \\
\text { syndiqués de la } \\
\text { CTCC/CSN par } \\
\text { rapport au total des } \\
\text { syndiqués } \\
\text { québécois }\end{array}$ \\
\hline 1901 & 74 & & 62 & 136 & \\
\hline 1911 & 190 & & 38 & 228 & \\
\hline 1921 & 334 & 120 & 38 & 492 & 24,0 \\
\hline 1931 & 286 & 121 & 84 & 491 & 21,6 \\
\hline 1940 & 306 & 239 & 153 & 698 & 33,1 \\
\hline 1951 & 459 & 439 & 228 & 1126 & 31,4 \\
\hline 1961 & 725 & 469 & 410 & 1606 & 24,6 \\
\hline 1970 & & & & 2665 & 29,2 \\
\hline 1976 & 904 & 1083 & 1437 & 3424 & 18,8 \\
\hline
\end{tabular}

28 Voir note 16

29 J. Rouillard, Les syndicats nationaux au Québec, op. cit., 240-250.

30 Le total comprend également des syndicats nationaux et indépendants. Gazette du Travail, sept. 1901 à mai 1902; Labour Organizations in Canada (1911): 94 et 104; (1921): 249, 268 et 272; (1931): 220 et 243; (1940): 29 et 181; (1951): 24 et $69 ;(1961)$ : XII et 1 à $64 ;(1971)$ : XX et 1 à 98; F. Delorme et G. Lassonde, op . cit., 13. 
Pendant les trente premières années du siècle, les «internationaux» regroupent environ les deux tiers des syndicats. Cette formidable expansion provoque la création par le clergé de syndicats catholiques destinés à maintenir la classe ouvrière dans le giron de l'Église. Mais leur développement s'avère beaucoup plus difficile que ne le prévoyaient ses propagateurs. Ce n'est finalement que dans les années trente, pour les raisons données plus haut, que le syndicalisme catholique connaît un taux de croissance supérieur aux «internationaux». Il réussit à réduire leur proportion de syndicats dans la province à $45 \%$ environ, pourcentage qu'ils maintiennent jusqu'à tout récemment.

Il ne faut pas croire que les «internationaux» à cette époque recrutent en majorité des travailleurs anglophones. Bien qu'il soit difficile de trouver des données précises à ce sujet, un observateur attentif de la scène syndicale, Alfred Charpentier, estime en 1918 que les deux tiers $(68 \%)$ des syndiqués internationaux à Montréal sont francophones ${ }^{31}$. C'est plausible car on retrouve à l'époque la majorité des organisations internationales dans l'industrie de la construction et le transport ferroviaire où les francophones constituent la grande majorité de la maind'oeuvre. Comme la présence «internationale» déborde la région métropolitaine, la proportion de francophones dans les rangs «internationaux» à l'échelle du Québec est sûrement supérieure à l'estimation de Charpentier pour Montréal. Personnellement, nous pensons que les non-francophones constituent tout au plus $25 \%$ des syndiqués internationaux au Québec depuis 1911. On a donc tort d'identifier les «internationaux» aux anglophones et la CTCC aux francophones.

Les données sur l'allégeance syndicale (Tableau IV) reposent sur l'affiliation des syndicats et non sur les effectifs de chacun des groupes syndicaux (sauf pour la CTCC/CSN). Une ventilation selon l'effectif est davantage révélatrice de l'importance de chacun de ces groupes. Malheureusement, les publications fédérales ne nous fournissent pas de données aussi désagrégées par province. Il est cependant possible de parvenir à des approximations valables. Comme les syndicats catholiques recrutent à peu près uniquement leurs membres au Québec, nous avons pu déterminer le pourcentage de syndiqués qu'ils représentent (voir le Tableau IV) en mettant en rapport leur membership avec les effectifs totaux du Québec. Pour les «internationaux» l'exercice est plus périlleux. L'affiliation aux centrales qui les regroupent au Québec (FPTQ, FUIQ, FTQ) est facultative, de sorte qu'on ne peut établir de relation directe entre leurs effectifs et le membership international au Québec. Sous toute réserve, il nous apparaît que ces effectifs sont probablement assez similaires à la proportion de syndicats qu'ils réunissent dans la province. On peut raisonnablement le penser car la relation est positive dans le cas de la CTCC/CSN. 1918), 15 . 
La syndicalisation pendant la dernière guerre et les années cinquante permet aux «internationaux» de connaître un taux de croissance supérieur aux syndicats catholiques. La proportion de syndiqués détenue par la CTCC passe de $31,4 \%$ à $24,6 \%$ de 1951 à 1961 . Le recrutement difficile de nouveaux adhérents explique en partie la déconfessionnalisation de la Centrale en 1960. On espérait ainsi se montrer plus accueillants pour les non-catholiques dans les grandes entreprises montréalaises $^{32}$.

Mais c'est surtout à la faveur de l'organisation du secteur public et parapublic que la CSN reprend le terrain perdu. Ses effectifs qui doublent dans les années soixante, lui permettent de regrouper en 1970 le tiers $(29,2 \%)$ des syndiqués québécois, un nouveau sommet pour la Centrale. Puis, surviennent le schisme de 1972 (fondation de la Centrale des Syndicats démocratiques) et le départ d'importants syndicats, ce qui entraîne une hémorragie de 70000 membres $^{33}$. En 1976, la CSN détient $18,8 \%$ des syndiqués au Québec contre 9,6 pour la CEQ et 4,7 pour la $\mathrm{CSD}^{34}$.

Pour leur part, les «internationaux» ne réussissent pas à garder leur proportion traditionnelle de syndiqués, qui passe de $42 \%$ à $37,2 \%$ de 1972 à $1976^{35}$. Le ralentissement économique des dernières années les frappe d'autant plus durement qu'ils sont surtout présents dans le secteur manufacturier. Le tertiaire privé où se dirige la main-d'oeuvre est difficile à organiser alors qu'ils sont absents du secteur public. Ces facteurs laissent prévoir un affaiblissement graduel du syndicalisme international tant au Québec qu'au Canada.

La FTQ, à laquelle sont affiliés ces syndicats, subit moins qu'on ne pourrait le croire les contrecoups de cette évolution. En effet, le recul «international» est compensé par l'accroissement de ses affiliés provenant des fédérations nationales (pancanadiennes), tel le Syndicat canadien de la fonction publique du Canada ou l'Alliance de la fonction publique du Canada. Depuis vingt ans, ces syndicats nationaux connaissent une forte expansion. Ainsi, la FTQ devient de moins en moins le porte-parole du syndicalisme international et davantage la voix du syndicalisme pancanadien au Québec.

\section{III) Le mouvement de grèves}

La propension à la grève apparaît un moyen éminemment précieux de mesurer le militantisme des travailleurs québécois. «Les grèves, écrit Robert Goetz-Girey, ne sont qu'un symptôme des conflits collectifs qui opposent employeurs et salariés... Mais elles en sont les formes les plus

\footnotetext{
32 J. Rouillard, Histoire de la CSN, op. cit., 179.

Ibid., 219.

34 F. Delorme et G. Lassonde, op. cit., 13.

35 Ibid., et Annuaire du Québec, (1977-1978): 735.
} 
apparentes, les plus «régulières» et surtout les seules qui puissent être analysées et mesurées avec une précision relative.» ${ }^{36}$ Pour le Québec, cette mesure est possible grâce encore une fois aux compilations du ministère fédéral du Travail. La publication annuelle depuis 1901 de Grèves et lock-out au Canada permet une continuité statistique sur la fréquence des grèves et lock-out et la perte des jours ouvrables entraînée par les conflits de travail (voir l'annexe pour une évaluation de cette source).

Nous comparons encore une fois le Québec à l'Ontario afin d'obtenir un point de repère et nous ne nous intéressons, dans le cadre de cet article, qu'aux seules données en jours-personne perdus. Cet indice s'avère le plus représentatif ${ }^{37}$ car il tient compte à la fois de la durée de la grève et du nombre de grévistes impliqués. Il est le produit de la multiplication du nombre de travailleurs directement impliqués dans un conflit par la durée de ce conflit. Pour rendre plausible la comparaison entre deux provinces qui ont des populations actives différentes, nous avons eu recours à un indice qui exprime le nombre moyen de jours ouvrables perdus annuellement par 100 personnes ayant un emploi rémunéré (population active non agricole) ou par 100 salariés lorsque cette donnée est disponible pour le Québec (1941-1980). Cet indice permet de comparer le poids réel des grèves et lock-out entre des régions et des périodes différentes.

\section{a) Période 1901-1941}

Dans son étude sur les conflits industriels au Canada, Stuart Jamieson constate qu'au début du siècle, les grèves les plus importantes dans le secteur manufacturier surviennent au Québec. «La province, écrit-il, est un endroit majeur d'agitation ouvrière et de conflits avant la Première Guerre mondiale. ${ }^{38}$ De toutes les provinces du Canada, seule la Colombie britannique connaît un niveau de grève supérieur. Ce phénomène l'étonne d'autant plus que ses lectures lui ont appris que le mouvement ouvrier dans la province francophone était docile et «dominé par le clergé» ${ }^{39}$.

Le niveau de grève, en effet, est élevé au Québec au début du siècle compte tenu de la faible population ouvrière.

Aussi surprenant que cela puisse paraître, il faudra attendre les années soixante pour que notre indice de l'importance des grèves dépasse celui de la période 1911-1921.

\footnotetext{
36 Robert Goetz-Girey, Le Mouvement des grèves en France (Paris, Sirey, 1965), 11.

37 John Vanderkamp, «Economic Activity and Strikes in Canada», Industrial Relations, 9, 2 (février 1970): 221.

38 Stuart M. Jamieson, Times of Trouble: Labour Unrest and Industrial Conflict in Canada, 1900-1966 (Ottawa, Information Canada, 1971), 86 (Étude no 22 pour la Commission Woods).

39 Ibid., 86.
} 


\begin{tabular}{|c|c|c|c|}
\hline \multicolumn{4}{|c|}{$\begin{array}{c}\text { TABLEAU V } \\
\text { NOMBRE MOYEN DE JOURS-PERSONNE PERDUS ANNUELLEMENT }{ }^{40} \text { AU } \\
\text { QUÉBEC PAR RAPPORT À LA POPULATION ACTIVE NON AGRICOLE, } \\
(1901-1960)\end{array}$} \\
\hline & $\begin{array}{l}\text { Jours-personne perdus } \\
\text { (moyenne annuelle) } \\
\text { (1) }\end{array}$ & $\begin{array}{c}\text { Population active } \\
\text { non agricole } \\
\text { (moyenne annuelle) } \\
\text { (2) }\end{array}$ & $1 / 2 \times 100$ \\
\hline $\begin{array}{l}1901-1911 \\
1911-1921 \\
1921-1931 \\
1931-1941 \\
1941-1950 \\
1951-1960 \\
1961-1970\end{array}$ & $\begin{array}{rr}80 & 329 \\
203 & 064 \\
96 & 409 \\
78 & 740 \\
282 & 526 \\
367 & 508 \\
978 & 911\end{array}$ & $\begin{array}{r}382300 \\
506500 \\
678980 \\
864300 \\
1048200 \\
1550700 \\
1 \quad 841800\end{array}$ & $\begin{array}{r}21,0 \\
40,0 \\
14,2 \\
9,1 \\
26,9 \\
23,7 \\
53,1\end{array}$ \\
\hline
\end{tabular}

Pendant les deux premières décennies du siècle, le mouvement des grèves est réparti très inégalement. Le plateau atteint entre 1901 et 1916 s'élève brusquement pendant la guerre et l'après-guerre (1917-1922). En terme de jours ouvrables perdus, l'année 1919 est un record dépassé uniquement en 1966 avec les grèves du secteur public et parapublic. C'est dire l'intensité des conflits qui sont survenus. Rappelons que le taux de syndicalisation aussi connaît une forte hausse à ce moment. L'effervescence ouvrière est reliée surtout à la forte poussée inflationniste qui sévit à la fin de la guerre (les prix doublent de 1916 à 1920).

Comme on l'aura noté, le mouvement ouvrier subit fortement les effets des cycles économiques puisque la négociation collective porte principalement sur des questions salariales. La majorité des conflits de travail au Canada résultent de désaccords sur les augmentations de salaire ou de questions qui y sont reliées ${ }^{41}$. D'autres facteurs aussi d'ordre politique, psycho-social ou socio-culturel interviennent pour influer sur le mouvement des grèves ${ }^{42}$. Mais comme ces facteurs sont difficilement quantifiables, il est malaisé d'en mesurer la portée. Dans une étude aussi sommaire que la nôtre, nous nous contenterons de les signaler au passage.

40 Je remercie Guy Bélanger pour la compilation de certaines données. BFS, Tendances occupationnelles, op . cit., 10 et 11 . Voir annexe. La moyenne annuelle de la population active non agricole est approximative et établie à partir de deux recensements décennaux. Pour les grèves: Canada, Department of Labour, Strikes and Lock-out in Canada, 1901-1916 (Ottawa, 1918); Gazette du Travail, 1916-1960.

41 Stuart M. Jamieson, Industrial Conflict in Canada 1966-1975 (Conseil économique du Canada, 1979), 6 (Discussion Paper no 142).

42 Ibid., 6; John Vanderkamp, op . cit., 228; Albert Rees, «Industrial Conflict and Business Fluctuations», dans A. Kornhauser, R. Dubin et A. Ross, Industrial Conflict (New York, McGraw Hill, 1954), 220; U.A. Frank, M.J. Kelly et B.D. MacNaughton, «Legislative change and strike activity, 1926-1974», Relations Industrielles, 37,2 (1982): 267-283. 
Tous les auteurs sont d'accord pour dire que l'inflation exerce une influence positive sur la fréquence et l'intensité des grèves ${ }^{43}$. C'est pourquoi il n'est pas surprenant qu'après la Première Guerre, avec une inflation aussi forte, notre indicateur des grèves ait atteint un sommet qui n'a été dépassé que très récemment. D'autres éléments expliquent aussi la flambée des grèves: la prospérité de guerre, le faible chômage et un climat social propice au changement dans tous les pays industrialisés (révolution russe, grève de Winnipeg, montée du Parti socialiste aux États-Unis, etc). D'ailleurs ces pays observent aussi une recrudescence des grèves pendant ces années.

Au cours des années vingt et trente, la propension à la grève diminue à cause du chômage engendré par la récession de 1921 et par la crise économique de 1929. La force revendicative des salariés s'en trouve diminuée alors que la résistance patronale augmente. Les employeurs sont certains d'obtenir toute la main-d'oeuvre requise et les salariés craignent de perdre leur emploi. On comprend qu'ils ne soient pas alors enclins à faire la grève ou à joindre un syndicat.

Pendant les quarante premières années du siècle, certaines grèves importantes ont gonflé les statistiques annuelles. Ce sont les grèves des débardeurs de Montréal en 1903, de l'imprimerie en 1922, des ouvriers de la chaussure en 1903, 1914 et 1926, du textile en 1908, 1917 et 1937 ainsi que celles des ouvriers de la confection en 1912, 1917, 1926 et 1934. L'industrie légère à haute intensité de main-d'oeuvre est donc le théâtre de nombreux conflits de travail. Les grévistes sont presque tous canadiens-français dans la chaussure et le textile alors que dans la confection, ils appartiennent majoritairement à la communauté juive ${ }^{44}$.

\begin{tabular}{|c|c|c|c|c|}
\hline \multicolumn{5}{|c|}{$\begin{array}{c}\text { TABLEAU VI } \\
\text { NOMBRE MOYEN DE JOURS-PERSONNE PERDUS ANNUELLEMENT }{ }^{45} \\
\text { EN ONTARIO (1901-1941) }\end{array}$} \\
\hline & $\begin{array}{c}\text { Jours-personne } \\
\text { perdus } \\
\text { (moyenne annuelle) } \\
\text { (1) }\end{array}$ & $\begin{array}{l}\text { Population active } \\
\text { non agricole } \\
\text { (moyenne annuelle) } \\
\text { (2) }\end{array}$ & \multicolumn{2}{|c|}{$\begin{array}{l}\text { Jours-personne perdus } \\
\text { (moyenne annuelle) } \\
1 / 2 \times 100\end{array}$} \\
\hline $\begin{array}{l}1901-1911 \\
1911-1921 \\
1921-1931 \\
1931-1941\end{array}$ & $\begin{array}{l}118700 \\
246205 \\
134600 \\
123436\end{array}$ & $\begin{array}{r}565700 \\
753700 \\
932600 \\
1112800\end{array}$ & $\begin{array}{l}20,8 \\
32,6 \\
14,4 \\
11,0\end{array}$ & $\begin{array}{r}21,0 \\
40,0 \\
14,2 \\
9,1\end{array}$ \\
\hline
\end{tabular}

43 William D. Walsh, «Economic Conditions and Strike Activity in Canada», Industrial Relations, 14, 1 (février 1975): 53.

44 J. Rouillard, «Les travailleurs juifs de la confection à Montréal», Labour/Le Travailleur, 8/9 (aut.-printemps 1982): 253-259.

45 Pour le Québec, l'indice est calculé au tableau V. Même note qu'au tableau V. 
Comme le tableau ci-contre le démontre, l'Ontario ne manifeste pas un niveau de grève supérieur au Québec lorsqu'on tient compte de leur population active respective.

Notre indice de l'importance des grèves s'avère à peu près identique pour les années 1901-11 et 1921-41, mais, pour la décennie intermédiaire, le Québec devance la province voisine.

Encore une fois, l'interprétation d'une prétendue docilité des travailleurs québécois ne tient pas le coup. Il faudrait plutôt se demander pourquoi l'Ontario tire de l'arrière tant dans l'activité de grèves (19111921) que pour le niveau de syndicalisation. Stuart Jamieson a probablement la réponse quand il signale que l'industrie lourde en Ontario n'est pas organisée avant la fin des années trente et que le mouvement syndical dans cette province est dominé par les très conservateurs syndicats de métier ${ }^{46}$. Dans l'industrie légère par contre, surreprésentée dans l'économie du Québec ${ }^{47}$, le mouvement syndical fait une percée significative au début du siècle. Les syndicats catholiques s'efforcent d'en regrouper les travailleurs et ces industries sont le théâtre de conflits majeurs. Les différences dans le mouvement de grève entre les deux provinces tiendraient donc beaucoup à leurs structures économiques.

\begin{tabular}{|c|c|c|c|}
\hline \multicolumn{4}{|c|}{$\begin{array}{c}\text { TABLEAU VII } \\
\text { NOMBRE MOYEN DE JOURS-PERSONNE PERDUS ANNUELLEMENT } \\
\text { PAR } 100 \text { SALARIÉS AU QUÉBEC (1941-1980) }\end{array}$} \\
\hline & $\begin{array}{l}\text { Jours-personne perdus } \\
\text { (moyenne annuelle) } \\
\text { (1) }\end{array}$ & $\begin{array}{l}\text { Nombre de } \\
\text { salariés } \\
\text { (moyenne annuelle) } \\
\text { (2) }\end{array}$ & $1 / 2 \times 100$ \\
\hline 1941-1945 & 161012 & 868400 & 18,5 \\
\hline $1946-1950$ & 404041 & 944600 & 42,7 \\
\hline $1951-1955$ & 399566 & 1117200 & 35,7 \\
\hline $1956-1960$ & 335451 & 1313400 & 25,5 \\
\hline $1961-1965$ & 484248 & 1422800 & 34,0 \\
\hline $1966-1970$ & 1473574 & 1700840 & 86,6 \\
\hline $1971-1975$ & 2180620 & 1924680 & 113,3 \\
\hline $1976-1980$ & 3350440 & 2180800 & 153,6 \\
\hline
\end{tabular}

46 Stuart M. Jamieson, Times of Trouble..., op. cit., 253. Voir aussi Irving Abella, Nationalism, Communism, and Canadian Labour (Toronto, University of Toronto Press), 5-8, 54, 86. 7 Albert Faucher et Maurice Lamontagne, «L'histoire du développement industriel au Québec», dans M. Rioux et Y. Martin, op cit., 268-271.

48 Grèves: Canada, Ministère du Travail, Grèves et lock-out au Canada, 1945-1980; La Gazette du Travail, 1941-1955. Salariés: BFS, Canadian Labour Force Estimates, 1931-1945, Reference Paper 23, 18; The Labour Force, 1945-1955: 98; 1956-1961; Estimations du nombre de salariés par province et par industrie, 1961-1982. 


\section{b) Période 1941-1980}

Le redressement économique de la Deuxième Guerre s'est accompagné d'une recrudescence du mouvement de grèves. Le plein emploi place les syndicats dans une meilleure position pour rattraper les pertes subies au cours des années antérieures. Les conflits sont nombreux, mais de courte durée, car le gouvernement établit des mécanismes obligatoires de conciliation dans l'industrie de guerre. C'est pourquoi, en terme de jours ouvrables perdus, les grèves sont moins intenses pendant la guerre que durant les années ultérieures. (Voir Tableau VII)

L'intensité des grèves s'accroît après la guerre, d'autant plus que certains employeurs cherchent à récupérer les concessions accordées pendant le conflit alors que d'autres s'ingénient à refuser la négociation collective. L'inflation d'après-guerre contribue également à rendre plus difficile les relations de travail ${ }^{49}$. Les conflits touchent surtout les entreprises du textile et de la confection, de même que l'industrie métallurgique et des métaux primaires, alors en pleine expansion et en voie de syndicalisation $^{50}$. L'année 1949 avec la grève de l'amiante contribue à gonfler les données en jours ouvrables perdus; elle est la grève la plus importante à survenir au Québec à cette date.

Malgré certaines grèves retentissantes comme celles de Louiseville (1952), Dupuis Frères (1952) et Murdochville (1957), les années cinquante ne voient pas de recrudescence particulière du mouvement de grèves. Les prix restent stables et la prospérité permet un accroissement marqué du salaire réel des travailleurs. Le vieillissement de la main-d'oeuvre peut expliquer aussi son conservatisme ${ }^{51}$. L'incidence des grèves tout comme le taux de syndicalisation se maintient à un niveau assez stable jusqu'en 1965.

Après cette date, l'intensité des conflits fait un bond spectaculaire: notre indice mesurant le poids réel des grèves est multiplié par trois au cours des quinze dernières années. C'est une progression étonnante qui, malheureusement, n'a pas fait l'objet d'études. Dans le cadre d'un article aussi général que le nôtre, on comprendra que nos explications demeurent superficielles.

Les conflits du secteur public et parapublic n'expliquent qu'en partie cette progression. Depuis qu'ils ont conquis le droit de grève en 1964 et 1965, ces employés ont été mêlés à plusieurs arrêts de travail qui ont pris des proportions de plus en plus importantes à mesure que les négociations se sont centralisées. À partir de 1972, ces négociations

49 Stuart M. Jamieson, Times of Trouble ..., op. cit., 296

50 Richard Ouellet, «Les grèves: une analyse quantitative», dans J. Hamelin et F. Harvey, Les Travailleurs québécois, 1941-1971 (Cahiers de 1'Institut supérieur des Sciences humaines, Université Laval, 1976): 435 et suivantes.

${ }_{51} \quad$ Stuart M. Jamieson, Times of Trouble..., op. cit., 345-358. 
se déroulent en front commun pour la plupart des syndiqués (fonctionnaires, employés d'hôpitaux, enseignants, professionnels du gouvernement). Affrontant le gouvernement en nombre considérable, leurs arrêts de travail expliquent l'intensité du mouvement de grève pour certaines années: 1965, 1972, 1976 et 1979. Mais, au total, ces conflits ne représentent que 20\% des jours-personne perdus au Québec de 1965 à $1978^{52}$. Il faut donc chercher ailleurs, dans le secteur privé, les raisons de cette poussée du militantisme des travailleurs.

En tête de liste parmi ces facteurs, il y a la poussée inflationniste depuis 1974 alors que la croissance de l'indice des prix atteint des niveaux très supérieurs aux années précédentes. Cette situation suscite des incertitudes et de fortes exigences salariales qui se sont traduites par de longs et durs conflits de travail ${ }^{53}$. La réaction des travailleurs québécois est similaire à celle du reste du Canada et de la plupart des pays occidentaux qui voient les arrêts de travail se multiplier dans les années soixante-dix.

Toutefois, le Canada et l'Italie se situent au sommet des pays industrialisés pour l'incidence des grèves mesurée en jours ouvrables ${ }^{54}$. Dans une étude consacrée à l'ensemble canadien, Stuart Jamieson identifie plusieurs éléments d'explication qu'on peut appliquer au Québec. Nous retenons la décentralisation des structures de négociation et l'écart des échelles salariales en Amérique du Nord, la jeunesse des travailleurs dans les années soixante, la croissance des profits des entreprises et l'instabilité de la structure économique du Canada à cause de sa spécialisation dans l'exportation de matières premières et de biens semifinis ${ }^{55}$. Il faut remarquer enfin que, même à un niveau aussi élevé, les grèves et lock-out représentent moins de $1 / 2 \%$ des journées-personne d'emploi au Canada pour 1966-1975 ${ }^{56}$.

Poursuivant notre comparaison avec l'Ontario, on remarque, selon notre indicateur, une incidence des grèves supérieure dans cette province de 1941 à 1960 et un niveau similaire pour les quinze années suivantes. (Voir Tableau VIII)

Pendant la dernière guerre et les années subséquentes, des conflits majeurs ébranlent l'industrie lourde ontarienne (automobile, acier primaire, fonte et affinage des métaux non ferreux $)^{57}$. C'est pourquoi notre indicateur des grèves dans cette province dépasse largement celui du Québec jusqu'en 1960.

\footnotetext{
52 André Beaucage, Idéologie, solidarité et politique salariale: l'expérience des fronts communs du secteur public québécois, de 1971 à 1975 (Ph.D., relations industrielles, Université de Montréal, 1981), 12.

53 Stuart M. Jamieson, Industrial Conflict..., op. cit., 29 et 64; W.D. Walsh, op. cit., 53.

54 Stuart M. Jamieson, Industrial Conflict..., op. cit., 4.

55 Ibid., 70 p.

56 Ibid., 58.

57 Stuart M. Jamieson, Times of Trouble..., op. cit., 284-286, 298 et 372.
} 


\begin{tabular}{|c|c|c|c|c|}
\hline \multicolumn{5}{|c|}{$\begin{array}{c}\text { TABLEAU VIII } \\
\text { NOMBRE MOYEN DE JOURS-PERSONNE PERDUS ANNUELLEMENT }{ }^{58} \\
\text { PAR 100 SALARIÉS EN ONTARIO (1941-1980) }\end{array}$} \\
\hline & $\begin{array}{l}\text { Jours-personne } \\
\text { perdus } \\
\text { (moyenne annuelle) } \\
\text { (1) }\end{array}$ & $\begin{array}{l}\text { Nombre de salariés } \\
\text { (moyenne annuelle) } \\
\text { (2) }\end{array}$ & \multicolumn{2}{|c|}{$\begin{array}{c}\text { Jours-personne perdus } \\
\text { (moyenne annuelle) } \\
1 / 2 \times 100\end{array}$} \\
\hline $\begin{array}{l}1941-1945 \\
1946-1950 \\
1951-1955 \\
1956-1960 \\
1961-1965 \\
1966-1970 \\
1971-1975 \\
1976-1980\end{array}$ & $\begin{array}{r}407364 \\
712270 \\
787647 \\
739736 \\
704836 \\
2737350 \\
2185730 \\
1991616\end{array}$ & $\begin{array}{llll}1 & 114 & 600 \\
1 & 329 & 000 \\
1 & 530 & 800 \\
1 & 822 & 200 \\
2 & 075 & 600 \\
2 & 539 & 900 \\
2 & 934 & 380 \\
3 & 438 & 900\end{array}$ & $\begin{array}{r}36,5 \\
53,5 \\
51,4 \\
40,5 \\
33,9 \\
100,7 \\
74,4 \\
57,9\end{array}$ & $\begin{array}{r}18,5 \\
42,7 \\
35,7 \\
25,5 \\
34,0 \\
86,6 \\
113,3 \\
153,6\end{array}$ \\
\hline
\end{tabular}

Après cette date, l'intensité des grèves est comparable dans les deux provinces, sauf pendant l'année 1976, qui rompt l'équilibre en faveur du Québec. Deux événements se sont conjugués pour faire de 1976 une année record en terme de jours ouvrables perdus (6 456 650): des grèves d'envergure dans l'industrie de la construction et les conflits $\mathrm{du}$ front commun dans le secteur public ${ }^{59}$. Mais si on excepte cette année-là, l'évolution de l'incidence des grèves est à peu près identique dans les deux provinces.

Toutefois, il y a une différence dans le secteur économique le plus touché (1960-1975): les industries lourdes citées plus haut viennent en tête de liste en Ontario $(37,5 \%)$ tandis que le secteur public et parapublic accapare la proportion la plus forte de jours ouvrables perdus au Québec $(21,3 \%)^{60}$. Les travailleurs de chacun de ces groupes industriels sont devenus le fer de lance du mouvement syndical dans l'une et l'autre province.

Notre recherche démontre que le militantisme des travailleurs québécois, estimé selon le niveau de syndicalisation et le mouvement des grèves, se compare à celui de la province voisine, et ce, depuis le début du siècle. L'industrialisation et le développement du capitalisme se sont traduits par le développement d'une classe ouvrière aussi éveillée aux problèmes industriels que ne pouvaient l'être les travailleurs ontariens. La thèse du supposé silence ou du retard du mouvement ouvrier qué-

58 Mêmes notes qu'au tableau VII.

59 F. Delorme, G. Lassonde et L. Tremblay, Grèves et lock-out au Québec, 1966-1976. Quelques précisions sur les modes de compilation (Québec, Ministère du Travail et de la Maind'oeuvre, 1977), 31.

60 Stuart M. Jamieson, Industrial Conflict..., op. cit., 38. 
bécois n'est pas fondée, car sa présence autonome s'affirme dans notre société bien avant la grève de l'amiante et les années cinquante. La conscience ouvrière se développe graduellement depuis le début du siècle en parallèle avec celle du reste du continent nord-américain. Le nationalisme tel que défini par Dofny et Rioux n'a donc pas fait obstacle au développement de la classe ouvrière au Québec.

S'il est quelques différences dans l'évolution du syndicalisme et de l'activité de grèves entre le Québec et l'Ontario, c'est à la fois du côté de l'économie et du socio-politique qu'il faut chercher les principaux éléments d'explication. Les industries de biens de consommation (textile, chaussure confection) qui s'implantent davantage au Québec et qui connaissent une phase d'expansion jusqu'à la récession de 1921 sont perméables à la syndicalisation au début du siècle, du moins pour une portion significative de leurs employés. Les syndicats internationaux s'assurent l'adhésion des ouvriers juifs de la confection alors que les syndicats nationaux et catholiques regroupent les ouvriers de la chaussure et, avec plus de difficultés et pour de courtes périodes, ceux $\mathrm{du}$ textile ${ }^{61}$. Leur militantisme s'affirme également à l'occasion de nombreux conflits qui touchent un grand nombre de travailleurs, car les arrêts de travail sont souvent simultanés dans plusieurs entreprises.

Par contre, en Ontario, où l'on observe une spécialisation dans l'industrie lourde, la majorité des travailleurs de ces industries ne sont pas syndiqués avant la fin des années trente et leurs arrêts de travail sont peu fréquents. Il faut attendre leur organisation par les syndicats industriels dans les années quarante et cinquante pour assister à une croissance plus rapide qu'au Québec du taux global de syndicalisation et du niveau de grève. Pendant ces deux décennies, les industries légères au Québec, surtout la chaussure et la confection, n'apportent plus la même contribution au mouvement de grèves.

Pour les années plus récentes, le militantisme des travailleurs de l'industrie lourde permet toujours à l'Ontario de maintenir un écart favorable dans le secteur privé. Mais la syndicalisation et la combativité des travailleurs du secteur public et parapublic permettent au Québec de rejoindre et même de dépasser les indicateurs de la province voisine.

Un facteur d'ordre socio-politique, la Révolution tranquille, est intervenu pour créer un contexte favorable à la syndicalisation, à la mobilisation et à la politisation du mouvement syndical. En effet, les changements sociaux profonds des années soixante aiguisent les aspirations vers une société plus juste et plus égalitaire. L'extension du rôle

61 J. Rouillard, Les travailleurs du coton au Québec 1900-1915 (Montréal, PUQ, 1973), 79-105; «Les travailleurs juifs...,» op . cit., 253-259; «Implantation et expansion de l'Union internationale des travailleurs en chaussures au Québec de 1900 à 1940», RHAF, 36, 1 (juin 1982): $75-105$. 
de l'État au même moment draîne, vers le tertiaire public, de jeunes travailleurs désireux de relever les conditions de travail moyenâgeuses du secteur public et de bâtir une société foncièrement différente. Évidemment, l'Ontario n'a pas vécu ce cheminement, et c'est pourquoi le tertiaire public y est moins bien syndiqué, plus conservateur et moins politisé.

\section{ANNEXE \\ Précisions sur les sources et la méthodologie}

\section{Taux de syndicalisation}

Les données recueillies par le ministère du Travail proviennent des déclarations faites par les syndicats eux-mêmes sans vérification plus approfondie $^{62}$. La même méthode de compilation est suivie aux ÉtatsUnis par le Bureau of Labor Statistics.

Les données sur l'affiliation syndicale sont sans doute assez fiables, mais celles qui portent sur le nombre de syndicats sous-évaluent leur nombre réel parce qu'on exclut les syndicats locaux indépendants groupant moins de 50 membres et que certains syndicats ne précisent pas la province où ils exercent leur activité. Cette dernière omission qui représente 6 pour cent de l'ensemble des effectifs en 1921 diminue en importance par la suite, pour disparaître après 1948.

Cette lacune statistique a aussi pour conséquence de sous-évaluer les effectifs syndicaux. Mais on a plusieurs raisons de croire en revanche que les syndicats dans leurs déclarations ont tendance à surestimer le nombre réel de syndiqués. Ils englobent des adhérents retraités, en chômage ou qui ne sont pas en règle afin d'afficher une force supérieure à la réalité. Dans quelle mesure ces données sont-elles gonflées; il est bien difficile de le déterminer dans l'état actuel de la recherche. Il est intéressant de signaler qu'aux États-Unis, un chercheur, Leo Troy, a effectué une étude sur les effectifs syndicaux à partir des rapports financiers des principaux syndicats (1933-62) et a comparé ses résultats avec les données établies par le Bureau of Labor Statistics. L'écart moyen était de l'ordre de 10 pour cent supérieur en faveur du $B L S^{63}$. On peut penser qu'une marge d'erreur semblable s'applique probablement aussi au Canada. Elle serait plus importante dans les premières décennies,

62 Voir pour plus de renseignements: Canada, Ministère du Travail, Croissance du syndicalisme au Canada, 1921-1967 (1970): 56-58; J.K. Eaton, op. cit., 179-190.

63 Leo Troy, op cit., 110. 
mais diminuerait par la suite à mesure que les méthodes de collecte des données se seront affinées.

D'autre part, les enquêtes excluent certains groupes syndicaux parce qu'ils ne correspondent pas à la définition officielle du ministère du Travail. Ainsi, pour le Québec, les syndicats catholiques sont écartés des compilations globales jusqu'en 1927, probablement parce qu'on les assimile à des associations religieuses. Nous avons fait la correction au tableau I pour 1916, 1921 et 1926 (effectifs corrigés des syndicats catholiques: $1916 ; 3500,1921 ; 23400,1926 ; 15300)^{64}$.

De même, les statistiques n'englobent pas les organisations de policiers, d'enseignants, d'infirmières et d'ingénieurs, à moins qu'elles ne soient affiliées à une centrale syndicale. Les recenseurs jugent que ces emplois ne comptent pas une proportion assez importante de leurs effectifs assujettis à la législation sur les relations du travail. Cette définition nous est apparue trop restrictive. Aussi, nous utilisons, à partir de 1966, les statistiques compilées par J.-K. Eaton, qui incluent les associations de fonctionnaires, d'infirmiers et d'enseignants ${ }^{65}$.

Autre problème relatif aux sources: certains syndicats ne déclarent pas leurs effectifs au ministère du Travail. Pour les deux provinces, ils sont 30 à 40 pour cent d'entre eux à négliger de le faire de 1911 à 1926; 20 pour cent environ au cours des deux décennies suivantes, et 10 pour cent dans les années cinquante et soixante. Pour palier à cette lacune importante, nous appliquons la règle de trois aux années 1911 à 1961 des tableaux I, II et III. Nous établissons la moyenne des adhérents de chaque syndicat ayant fait rapport et multiplions cette moyenne par le total des syndicats. On parvient ainsi à une estimation du nombre véritable de syndiqués pour chacune des années repères. Cette méthode comporte des faiblesses et surestime peut-être les effectifs syndicaux. En effet, on peut présumer que les grosses unités syndicales fournissent plus facilement des renseignements sur leurs effectifs que les unités plus faibles. Pour les premières décennies, la marge d'erreur pourrait être plus forte car plusieurs syndicats ne font pas rapport de leurs effectifs.

Mais, en mettant en relation ces estimations avec d'autres sources d'information, nous pensons qu'elles demeurent valables. Ainsi, le seul Conseil des métiers et du travail de Montréal (syndicats internationaux) revendique 25000 membres en 1912 alors que Le Monde Ouvrier évalue à 37928 en 1913 et à 34000 en 1916 les adhérents aux syndicats internationaux dans la métropole ${ }^{66}$. Toujours pour Montréal, Alfred

\footnotetext{
64 Voir notes du tableau $\mathrm{I}$.

65 J.K. Eaton, op. cit., 19, 47 et 183-190.

66 La Patrie, 2 août 1912, 2; Le Monde Ouvrier, 2 avril 1921, 3; André LeBlanc, Le Monde Ouvrier/The Labour World (1916-1926): an Analysis of Thought and a Detailed Index, (Thèse de DES (histoire), Université de Montréal, 1971), 7.
} 
Charpentier estime les effectifs syndicaux totaux à 40000 en $1918^{67}$. Ces évaluations comportent évidemment des exagérations, mais comme elles sont très inférieures à nos estimations, elles les rendent plausibles pour l'ensemble du Québec.

Nous sommes conscients que nos données demeurent des approximations, mais à l'intérieur d'une marge d'erreur raisonnable. Notre démarche nous apparaît utile car ces séries statistiques constituent des éléments essentiels à une bonne connaissance de l'évolution du syndicalisme québécois.

\section{Population active non agricole}

Il n'existe pas de données par province sur le nombre de salariés avant 1941. Quant à la population active (personnes ayant un emploi rémunéré), elle ne peut être obtenue que par décennie à partir des recensements du Canada dont les données sur l'occupation ont été uniformisées dans un bulletin spécial du recensement de $1941^{68}$. Nous avons établi le chiffre de la population active non agricole en soustrayant des totaux la population oeuvrant dans l'agriculture.

\section{Les grèves}

Dans la publication Grèves et lock-out au Canada, le ministère du Travail compile les grèves et lock-out au Canada depuis la fin de 1900. Il ne distingue pas les grèves des lock-out parce que ces derniers sont assez rares et qu'ils soni souvent difficiles à distinguer des grèves.

Les registres du ministère incluent toute cessation de travail affectant six travailleurs ou plus pendant une journée de travail ou plus; tout conflit qui cause la perte de dix jours ouvrables ou plus est automatiquement ajouté.

Les recenseurs s'efforcent d'avoir une recension complète de toutes les grèves si bien qu'il est pratiquement impossible qu'une grève importante n'ait pas été portée à leur connaissance. L'estimation du nombre de travailleurs impliqués et la durée du conflit est le résultat, nous dit la publication, de recherches minutieuses ${ }^{69}$. Seuls les travailleurs directement impliqués dans un conflit font partie de la recension.

La publication ventile ces données par province, mais isole les conflits débordant les cadres provinciaux de 1901 à 1956. Toutefois, comme ces conflits représentent en moyenne moins de un pour cent de jours ouvrables perdus, leur exclusion a peu de conséquences sur les

67 Archives de la CSN, Procès-verbal de la Journée sociale à la Villa Saint-Martin, 28 avril $1918,12$.

68 BFS, Tendances occupationnelles au Canada, 1901-1941, Bulletin no 0-6 du Recensement du Canada 1941 (98-1941, n. 33): 10 et 11.

69 La Gazette du Travail, mai 1942. 
données globales, sauf pour 1901-1915 et 1945-1951. Pour ces dernières années, la publication heureusement répartit, en bas de page, les données interprovinciales par province; nous avons fait les ajouts qui s'imposaient. Quant aux années 1901-1915, l'ampleur des conflits interprovinciaux $(23,3 \%$ des jours ouvrables perdus au Canada, surtout des grèves de cheminots) influent d'une manière significative sur les données provinciales. Mais comme nous n'avons pas les informations nécessaires pour les distribuer par province, nos données pour ces années aux tableaux V et VI sous-estiment donc l'intensité des grèves au Québec comme en Ontario.

Il en est de même de 1957 à 1980 car les conflits de juridiction fédérale (relevant du Code canadien du travail et de la fonction publique fédérale) sont classés sous une rubrique spéciale sans être distribués par province. Le rapport annuel du Centre de recherche et de statistiques sur le marché du travail (Québec) évalue les conflits de compétence fédérale à $8,6 \%$ des jours ouvrables perdus (1966 à 1980) ${ }^{70}$. Nous aurions pu utiliser ces données plus complètes pour le Québec, mais elles nous auraient empêché de faire une comparaison valable avec l'Ontario. Les données qui portent sur la période 1957 à 1980 sont donc sous-estimées d'environ 8\% pour le Québec et d'un peu plus pour l'Ontario, car les fonctionnaires fédéraux y sont plus nombreux.

Néanmoins, les lacunes statistiques qui viennent d'être signalées n'infirment en rien nos conclusions; au contraire, elles renforcent nos constatations à savoir que le niveau de grèves est élevé au début du siècle et que l'intensité des conflits fait un bond spectaculaire après 1965. 